\title{
Genetic Testing
}

National Human Genome Research Institute (NHGRI)

\section{Source}

National Human Genome Research Institute (NHGRI). Genetic Testing.

Genetic testing is the use of a laboratory test to look for genetic variations associated with a disease. The results of a genetic test can be used to confirm or rule out a suspected genetic disease or to determine the likelihood of a person passing on a mutation to their offspring. Genetic testing may be performed prenatally or after birth. Ideally, a person who undergoes a genetic test will discuss the meaning of the test and its results with a genetic counselor. 